\title{
Tezepelumab as an Emerging Therapeutic Option for the Treatment of Severe Asthma: Evidence to Date
}

This article was published in the following Dove Press journal:

Drug Design, Development and Therapy

\section{Zachariah L Dorey-Stein (D) Kartik V Shenoy}

Department of Thoracic Medicine and Surgery, Lewis Katz School of Medicine at Temple University, Philadelphia, PA, USA
Correspondence: Zachariah L Dorey-Stein Department of Thoracic Medicine and Surgery, Lewis Katz School of Medicine at Temple University, 340I North Broad Street, 7th Floor, Philadelphia, PA 19140, USA

$\mathrm{Tel}+\mathrm{I} 267-300-8047$

Fax + I 215-707-6867

Email zachariah.dorey-stein@tuhs.temple. edu
Abstract: Asthma is a complex heterogeneous disease defined by chronic inflammation of the airways. Patients present with wheezing, chest tightness, cough and shortness of breath. Bronchial hyperresponsiveness and variable expiratory airflow limitation are hallmark features. About $3.6-6.1 \%$ of patients, despite receiving high-dose inhaled corticosteroids (ICS) and a second controller medication, report persistent symptoms referred to as severe asthma. Uncontrolled severe asthma is associated with increased mortality, morbidity, diminished quality of life and increased health expenditures. The development of modern biological therapy has revolutionized severe asthma treatment. By targeting specific chemokines, asthma control has drastically improved, resulting in better quality of life, less emergency department visits and inpatient admissions, and decreased chronic systemic corticosteroid utilization. Despite these advances, there remains a subset of asthma patients who remain symptomatic with poor quality of life and heavy utilization of the healthcare system. Recently attention has been given to pharmaceutical therapy directed at receptors and cytokines on the epithelial layer of the lung referred to as "alarmins". Thymic stromal lymphopoietin (TSLP) is an interleukin-7-like receptor family found on the epithelial layer of the lung that releases a cytokine cascade inducing eosinophilic inflammation, mucus production and airflow obstruction in asthmatics. Tezepelumab is the first investigational monoclonal antibody that inhibits TSLP. Proof of concept study and phase IIb studies demonstrated reduced asthma exacerbations, improvement in quality of life, less decline in $\mathrm{FEV}_{1}$ and decrease in biochemical inflammatory markers in comparison to placebo. It is presently undergoing three phase III studies and an additional phase II study.

Keywords: tezepelumab, severe uncontrolled asthma, eosinophilic asthma, non-eosinophilic asthma, monoclonal antibody, biological therapy

\section{Introduction}

Asthma is a complex heterogeneous disease defined by chronic inflammation of the airways affecting more than 300 million people worldwide. ${ }^{1}$ Patients present with wheezing, chest tightness, cough and shortness of breath. Bronchial hyperresponsiveness and variable expiratory airflow limitation are hallmark features inducing airway damage and remodeling. The majority of patients are controlled with traditional inhaler therapy. However, 3.6-6.1\% of patients, despite receiving high-dose inhaled corticosteroids (ICS) and a second controller medication, report persistent symptoms referred to as severe asthma. ${ }^{2}$ Uncontrolled severe asthma is associated with increased mortality, morbidity, diminished quality of life and increased health expenditures. ${ }^{2}$ 
The development of modern biological therapy has revolutionized severe asthma treatment. By targeting specific chemokines, asthma control has drastically improved, resulting in better quality of life, less emergency department visits and inpatient admissions, and decreased chronic systemic corticosteroids utilization. The incomplete efficacy of current biological therapy is attributed to the agents incompletely treating the inflammatory pathway due to targeting chemokines downstream in the inflammatory cascade, and despite these advances there remains a subset of asthma patients who are severely uncontrolled.

Alternative treatment targets are warranted to treat patients with uncontrolled severe asthma who have not responded to present standard of care. Recently attention has been given to pharmaceutical therapy directed at receptors and cytokines on the epithelial layer of the lung referred to as "alarmins". Composed of thymic stromal lymphopoietin (TSLP), IL-33 and IL-25, high levels of these upstream cytokines are released from the epithelium in individuals with T-helper type (Th)-2 lymphocyte-high inflammation after exposure to irritants such as bacteria, viruses, allergens and environmental pollutants. Subsequently, they induce Th2 proinflammatory cytokines resulting in eosinophilic inflammation, mucus production and airflow obstruction (Figure 1). Due to their upstream location, alarmins have become a desired pharmaceutical target, as, hypothetically, by inhibiting one of these sites, the entire $\mathrm{Th} 2$ inflammatory cascade could be impeded.

Efforts to develop a monoclonal antibody to block IL25 have been limited, with 2 clinical trials completed to date. The first was a negative trial, and the second has not officially reported results. ${ }^{3}$ Several different agents have been developed with IL-33 as a target, but trials have had minimal success. Interim analysis of ECLIPSE phase II clinical trial of the monoclonal antibody etokimab, reported in fall of 2020, failed to show improvement in primary outcomes of reduction of nasal polyps and quality

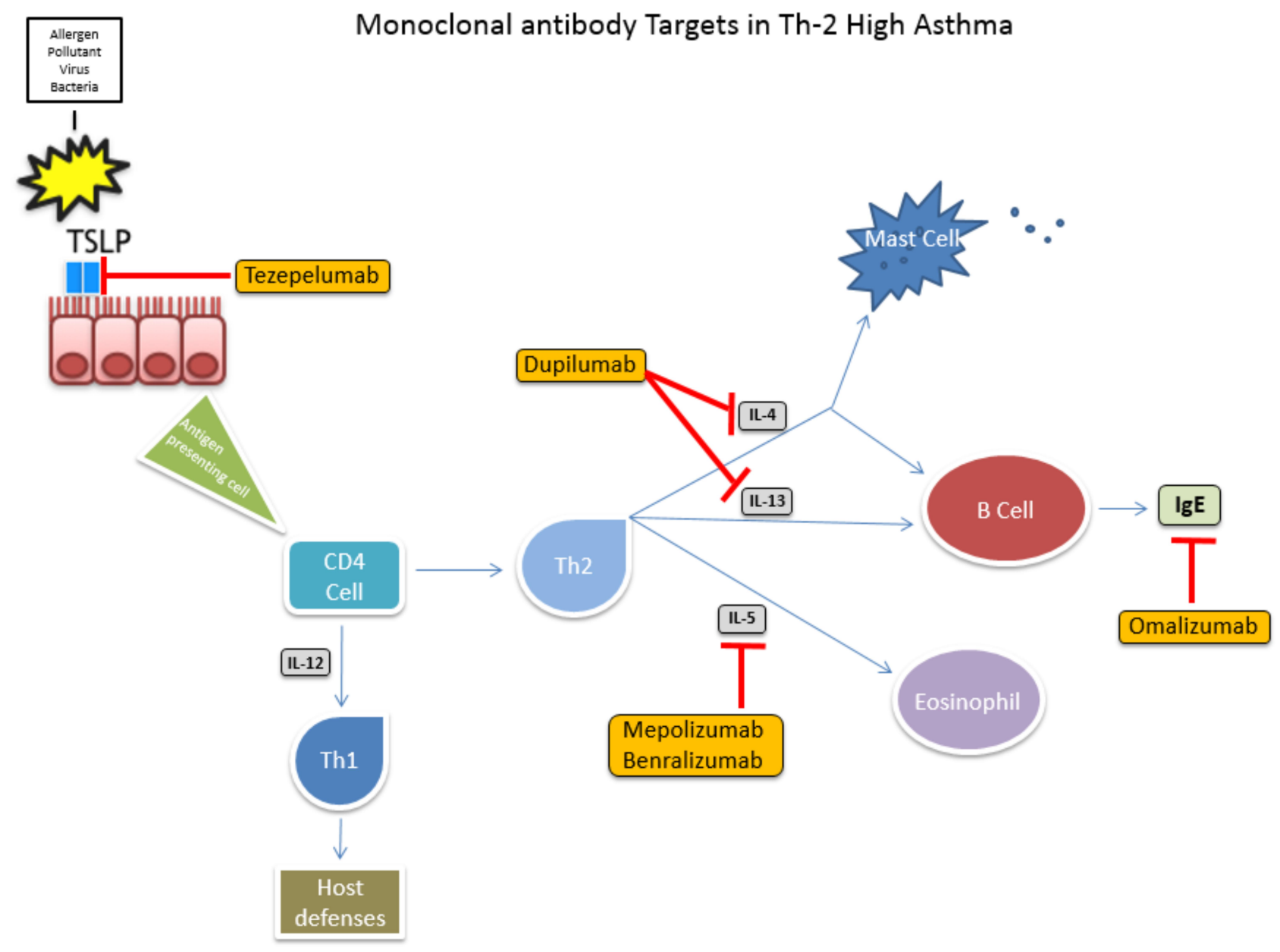

Figure I Monoclonal antibody targets in TH2-high asthma.

Abbreviations: TSLP, thymic stromal lymphopoietin; IL, interleukin; Th2, type 2 inflammation; ThI, type I inflammation; IgE, immunoglobulin E. 
of life, although it did note reduction in peripheral blood eosinophilia. ${ }^{4}$

In contrast, therapy directed at TSLP receptor inhibition has been much more favorable. Below we describe the pathophysiology of TSLP receptor-directed Th2 inflammation and review clinical trials that tezepelumab, a novel anti-TSLP receptor monoclonal antibody, has undergone as well as ongoing clinical trials.

\section{Pathophysiology}

Severe asthma encapsulates two predominant heterogeneous inflammatory endotypes, T-helper type (Th)-2 lymphocyteshigh and Th2-low. Some $50-70 \%$ of asthma patients are thought to have a Th2-high endotype. ${ }^{5,6}$ Th2-high is differentiated from Th2-low by the presence of eosinophilia in the sputum $(>3 \%)$ or peripheral blood $\left(>0.25 \times 10^{9} / \mathrm{L}\right){ }^{7}{ }^{7} \mathrm{Th} 2$ high endotype is driven by interleukin (IL)-3, IL-4, IL-5, IL9, IL-13, and granulocyte-macrophage colony-stimulating factor (GM-CSF) inducing eosinophil maturation, basophil recruitment and immunoglobulin E (IgE) production. Severe asthma, regardless of endotype, has strong associations with obesity, gastroesophageal reflux disease, depression and obstructive sleep apnea.

Asthma's diverse endotypes are attributed to complex interactions between an individual's genetic predisposition and environment resulting in abnormal sensitization to and responsiveness to airborne particles. After an initial exposure to an allergen, dendritic cells present the antigen to naïve $\mathrm{CD} 4+\mathrm{T}$ cells, which differentiate and form excessive allergen-specific Th2 lymphocytes. Th2 cells secrete a multitude of interleukins in a process referred to as sensitization. Subsequent allergen recognition and response occurs in the airways, inducing airway inflammation via inflammatory-cell recruitment, activation and mediator release. ${ }^{8}$ IgE binds allergens and synthesizes mediators releasing histamine, leukotrienes and cytokines. These chemokines induce, release and recruit inflammatory cells resulting in vascular permeability, smooth muscle contraction, smooth muscle hypertrophy, mucus production and hypersecretion in the lungs.

Type 2 inflammation is eosinophilic stimulated systemic airway inflammation that occurs in response to airborne irritants such as allergens, bacteria, virus, fungi and noxious inhalants. The cascade is initiated by epithelial cell exposure to environmental stimuli resulting in secretion of cytokines and chemotaxis of eosinophils, basophils and Th2 cells. Type 2 innate lymphoid cells are activated via IL-33 and TSLP and produce and activate Th2-cytokines. Following stimulation,
Th2-cytokines induce basophil recruitment (GM-CSF and IL-3), eosinophil maturation and differentiation (GM-CSF, IL-3, IL-5), mast cell maturation and differentiation (IL-3, IL-9, IL-13) and IgE (IL-4). ${ }^{9,10}$ The end result is mucous production, airway granulocyte activation and airway hyperreactivity. Chronic inflammation causes fixed obstruction in the airway of asthmatics due to airway remodeling.

Th2-low asthma comprises several different phenotypes including asthma associated with obesity, smoking, bacterial and viral infections as well as late-onset asthma. The pathophysiology of Th2-low asthma is not as well delineated as Th2-high asthma but is hypothesized to be secondary to neutrophilic inflammation via dysregulation of the Th17 pathway, a Th1 process, as well as unspecified Th2 pathways. At present, targeted treatment for Th2-low asthma is limited to dupilumab, an IL-4 $\alpha$ receptor blocker that inhibits IL-4 and IL-13 signaling. However, in clinical trials dupilumab has been shown to be more efficacious in patients with Th2 than Th1 inflammation. Rabe et al reported the greatest magnitude of AAER decline and $\mathrm{FEV}_{1}$ improvement in asthma patients with elevated blood eosinophilia ( $\geq 300$ cells $/ \mu \mathrm{L})$ and fractional exhaled nitric oxide $\left(\mathrm{FE}_{\mathrm{NO}}\right) \geq 50$ parts per billion $(\mathrm{ppb})$ versus placebo. ${ }^{11}$ Individuals with blood eosinophilia $\geq 150$ cells/ $\mu \mathrm{L}$ had a less robust response with no difference in AAER versus placebo, but improved $\mathrm{FEV}_{1}$, although not as pronounced as the previously mentioned cohort. No difference was noted in AAER or change in $\mathrm{FEV}_{1}$ in patients with blood eosinophils $<150$ cells $/ \mu \mathrm{L}$, indicative that the therapeutic functions predominantly along the Th2 pathway. Dupilumab has also demonstrated a reduction in chronic oral corticosteroids regardless of baseline blood eosinophil count; however, the magnitude of decline is greater in individuals with higher baseline blood eosinophil counts. ${ }^{12}$ Collectively, these findings demonstrate the need for novel targeted Th1 therapy.

TSLP is a pro-inflammatory epithelial pleiotropic IL-2 cytokine lining the surface of the skin, gastrointestinal tract and lungs that regulates immunity on barrier surfaces, tissue restoration and repair after an insult. It is also activated by chemokines and mediators associated with Th2 inflammation including basophils, mast cells, dendritic cells, IL-1, tumor necrosis factor (TNF)- $\alpha$, allergens, Toll-like receptor, bacterial and viral infections and NF- $\kappa \mathrm{B}$. Murine models have demonstrated increased production of TSLP due to uric acid release from allergens and double-stranded RNA viruses such as rhinovirus and respiratory syncytial virus (RSV). ${ }^{13-15}$ The receptor is upregulated by mechanical 
injuries, infection and other inflammatory cytokines including allergens, pollutants, bacteria and respiratory viruses. TSLP activates Th2 inflammation via activation of antigenpresenting cells, dendritic cells, mast cells, neutrophils and basophils inducing allergic inflammation and bronchial hyperresponsiveness. ${ }^{16,17}$ The epithelial lining of asthmatics with viral respiratory infections has higher levels of TSLP messenger rRNA and protein compared to healthy controls that correlates with increasing severity of asthma. $^{18-20}$

TSLP and Th2 chemokines were initially noted for their role in atopic diseases via dendritic cells. TSLP induces dendritic cell maturation to upregulate CD4 cells that differentiate into Th2 cells that initiate and augment the inflammatory cascade. Dendritic cells are also thought to cause stimulation via a positive feedback loop that further magnifies Th2 inflammation. TSLP has been hypothesized to induce sensitization and impede tolerance to allergens in the lung, further promoting the Th2 response. $^{21}$ TSLP upregulates basophil functions by permitting them to function as antigen-presenting cells and induces eosinophils, mast cells and basophil hematopoiesis via IL-3, further stimulating the Th2 inflammatory cascade.

Tezepelumab is the first investigational monoclonal antibody that inhibits human thymic stromal lymphopoietin receptor. It has been described as a "master switch" to obstruct atopic inflammation in murine models by preventing the initiation of the downstream interleukin-derived inflammatory cascade as indicated by reduction in $\mathrm{FE}_{\mathrm{NO}}$, blood eosinophils, IL-5, IL-13 and IgE. ${ }^{19,22}$ At present it is undergoing phase III trials.

\section{Completed Studies}

A proof of concept study to assess the effect of tezepelumab on 31 patients with mild allergic asthma was undertaken in 2014 (Table 1). ${ }^{23}$ Patients enrolled received tezepelumab $700 \mathrm{mg}$ or placebo for a one-hour infusion on days 1, 29 and 57. The primary endpoint was late asthmatic response measured 3 to 7 hours after allergen challenge on days 42 and 84 to evaluate the effect of tezepelumab in inhibiting reduction of the forced expiratory volume in 1 second $\left(\mathrm{FEV}_{1}\right)$. Secondary outcomes included reduction in inflammatory markers such as $\mathrm{FE}_{\mathrm{NO}}$, blood and sputum eosinophils and airway hyperresponsiveness.

Treatment with tezepelumab resulted in preserved lung function as noted by $34 \%$ less decline than placebo on day
$42(P=0.09)$ and $45.9 \%$ on day $84(P=0.02)$ in $\mathrm{FEV}_{1}$. Patients in the intervention arm also had a statistically significant decline in blood eosinophil count, sputum eosinophil count and $\mathrm{FE}_{\mathrm{NO}}$. There was no difference in serum IgE levels or statistical difference in adverse events.

PATHWAY, a phase IIb multicenter randomized double-blind dose-ranging study, enrolled 550 patients aged 18 to 75 years old with uncontrolled severe asthma to receive subcutaneous tezepelumab or placebo. ${ }^{24}$ Uncontrolled severe asthma was defined as an individual with symptoms despite taking a medium to high-dose inhaled corticosteroid and a long-acting beta-agonist (LABA). Patients were enrolled irrespective of their phenotype and followed for 52 weeks. The primary outcome was annualized asthma exacerbation rate. Asthma exacerbation was defined as worsening of asthma symptoms resulting in treatment with systemic glucocorticoids, doubling the dose of chronic glucocorticoids dose for 3 or more days, emergency department visit that resulted in glucocorticoid treatment or an admission due to asthma.

Enrollees were stratified by geographic location (Japan or the remainder of the world), baseline ICS dose (medium or high per GINA 2012 guidelines) and blood eosinophil count ( $\geq 250$ or $<250$ cells $/ \mu \mathrm{L}$ ). Randomization was in a $1: 1: 1: 1$ fashion to tezepelumab low dose (70 mg every 4 weeks), medium dose (210 mg every 4 weeks), high dose (280 mg every 2 weeks) or placebo. Annual exacerbation rates were $62 \%, 71 \%$ and $66 \%$ lower than in placebo $(P<0.001$ for each group comparison). Time to first asthma exacerbation was longer in the tezepelumab cohort. Prebronchodilator FEV improved statistically significantly in the intervention arms at 52 weeks and was observed as early as 4 weeks. Change in Asthma Control Questionnaire (ACQ)-6 score was statistically improved in all three intervention groups $(P=0.059$, $P=0.039$ and $P=0.02$ as authors chose a $P$ value and alpha level of 0.1 as statistically significant) although with Asthma Quality of Life Questionnaire (AQLQ)+12 score it was only statistically notable with the high-dose tezepelumab cohort. Inflammatory biomarkers downtrended significantly in each of the intervention arms. Post-hoc analysis stratified each group by blood eosinophil count ( $<400$ cells $/ \mu \mathrm{L}$ vs $\geq 400$ cells $/ \mu \mathrm{L}$ ) and confirmed statistically significant reduction in annual asthma exacerbation rate in tezepelumab recipients versus placebo regardless of baseline eosinophil count.

Three serious adverse events were attributed to the intervention drug: pneumonia and cerebrovascular accident in the same patient in the low-dose tezepelumab group and Guillain-Barré syndrome in the medium-do 
Table I

\begin{tabular}{|c|c|c|c|c|}
\hline Study Name & Phase & N & Primary Outcome & Secondary Outcome \\
\hline NCTOI 405963 & 1 & 31 & $\begin{array}{l}34 \% \text { improvement in } \mathrm{FEV} \text {, on day } 84(P=0.02) \text { in } \\
\text { tezepelumab recipients versus placebo }\end{array}$ & $\begin{array}{l}\text { Decrease in blood eosinophil count in intervention arm } \\
\text { Decrease in FENO in intervention arm }\end{array}$ \\
\hline PATHWAY & Ilb & 550 & AAER $62-71 \%$ versus placebo $(P<0.00 I)$ & $\begin{array}{l}\text { FEV }, 120-150 \mathrm{~mL} \text { improvement in intervention arms } \\
\text { versus placebo } \\
(P=0.002-0.015) \\
\text { ACQ-6 score statistically significant improvement in } \\
\text { higher-dose intervention arms }\end{array}$ \\
\hline NAVIGATOR & III & 1061 & AAER over 52 weeks & $\begin{array}{l}\text { Change in FEV, at } 52 \text { weeks } \\
\text { Change in AQLQ+12 at } 52 \text { weeks } \\
\text { Change in ACQ-6 at } 52 \text { weeks } \\
\text { Time to first asthma exacerbation } \\
\text { Change in annual exacerbation visit requiring emergency } \\
\text { room visit, urgent care evaluation or hospitalization } \\
\text { Change in } \mathrm{FE}_{\mathrm{NO}} \text {, lgE and peripheral blood eosinophilia at } 52 \\
\text { weeks }\end{array}$ \\
\hline SOURCE & III & 150 & $\begin{array}{l}\text { Percent reduction from baseline daily OCS dose without } \\
\text { losing asthma control from baseline to week } 48\end{array}$ & $\begin{array}{l}\text { AAER } \\
\text { Proportion of patients with cessation of OCS at week } 48 \\
\text { Proportion of patients with daily OCS } \leq 5 \mathrm{mg} \\
\text { at week } 48 \\
\text { Change in FEV } 1 \text { at } 48 \text { weeks } \\
\text { Change in AQLQ+ } 12 \text { at } 48 \text { weeks } \\
\text { Change in ACQ- } 6 \text { at } 48 \text { weeks } \\
\text { Change in FENO, lgE and peripheral blood eosinophilia at } 48 \\
\text { weeks }\end{array}$ \\
\hline DESTINATION & III & 966 & $\begin{array}{l}\text { Serious adverse events and adjusted adverse events } \\
\text { between tezepelumab and placebo }\end{array}$ & AAER from baseline to week 104 \\
\hline CASCADE & II & 116 & $\begin{array}{l}\text { Change from baseline in airway submucosal inflammatory } \\
\text { cells on bronchoscopy biopsies }\end{array}$ & $\begin{array}{l}\text { Change in reticular basement membrane thickness from } \\
\text { baseline at week } 28 \\
\text { Change in airway epithelial integrity from baseline } \\
\text { Change in airway submucosal inflammatory cells from } \\
\text { baseline }\end{array}$ \\
\hline
\end{tabular}

se tezepelumab cohort; however, no statistical difference in adverse events between tezepelumab and placebo was established.

Corren and co-workers stratified PATHWAY cohorts by baseline eosinophil counts of $<250$ cells $/ \mu \mathrm{L}$ vs $\geq 250$ cells $\mu \mathrm{L}$ and compared baseline inflammatory markers including $\mathrm{FE}_{\mathrm{NO}}$, IL-5, IL-13 and periostin, AAER, frequency of hospitalizations, ED visits and length of ICU and hospital stay following 52 weeks of tezepelumab or placebo. ${ }^{25,26}$ The investigators concluded that irrespective of baseline inflammatory markers AAER was reduced by $66 \%$ in pooled tezepelumab cohorts versus placebo. Pham and colleagues reported that tezepelumab pooled groups had statistically significant reduction in biomarker levels of IL-5, IL-13, periostin, thymus and activation-regulated chemokine (TARC), eosinophil count, $\mathrm{FE}_{\mathrm{NO}}$ and $\mathrm{IgE}$ versus placebo. ${ }^{27}$ Significant reduction in AAER, irrespective of baseline body mass index (BMI), has been reported, further reflecting tezepelumab efficacy, as well as decreased matrix remodeling through an unknown mechanism. $^{28,29}$

\section{Ongoing Studies}

Following the results of PATHWAY, PATHFINDER phase III program was created with two foundational phase III studies: NAVIGATOR and SOURCE. In addition, two other studies - DESTINATION, a phase III study, and CASCADE, a phase II study - will follow the conclusion 
of NAVIGATOR and SOURCE. Tezepelumab dosing selected for each of these studies was $210 \mathrm{mg} \mathrm{Q} 4 \mathrm{~W}$.

NAVIGATOR (NCT03347279) is a randomized, doubleblind, placebo-controlled study enrolling over 1000 adult (18-80 years old) and adolescent (12-17 years old) patients with severe asthma not controlled on a medium to high-dose ICS and at least one other asthma medication. The study design incorporates a 5-6-week screening period followed by a 52-week treatment period and a 12-week follow-up period. The primary endpoint is AAER. Secondary endpoints include effect of tezepelumab on lung function, asthma control and health-related quality of life. ${ }^{30}$ Preliminary results released in the fall of 2020 indicated that tezepelumab had achieved the primary endpoint of reducing AAER over 52 weeks. $^{31}$ Subgroup analyses of patients with baseline eosinophil counts of less than 300 cells $/ \mu \mathrm{L}$ and 150 cells/ $\mu \mathrm{L}$ also demonstrated statistically significant reductions in AAER. ${ }^{31}$

SOURCE is a randomized double-blind, placebocontrolled study enrolling 150 patients with severe asthma requiring medium to high-dose ICS and LABA bronchodilator therapy and chronic oral corticosteroids. Preliminary results released in December 2020 by the investigators stated that SOURCE failed to meet the primary endpoint of reducing daily oral corticosteroids while maintaining asthma control. ${ }^{32}$ Official results of SOURCE are pending at this time.

DESTINATION is a randomized, double-blind, placebo-controlled study that will enroll approximately 960 patients who have completed either of the above studies. The primary outcome is comparing exposure-adjusted rate of adverse events between the control and placebo arms. Lastly, CASCADE is a phase II randomized double-blind, placebo-controlled study investigating airway inflammation reduction. A total of 116 patients with inadequately controlled moderate to severe asthma despite receiving an ICS and one other asthma controller medication will be enrolled and followed over 40 weeks. The primary outcome is change in airway submucosal inflammatory cells found on bronchoscopic biopsies from baseline.

\section{Conclusion}

While phase III trials are still being completed, phase I and II trials strikingly concluded tezepelumab reduced asthma exacerbation rates, improved $\mathrm{FEV}_{1}$ and reduced type 2 inflammatory biomarkers compared to traditional bronchodilator therapy in individuals with high and low Th2 status with no increase in adverse reactions. At present multiple FDA-approved monoclonal antibody agents are available to treat patients with uncontrolled allergic and eosinophilic asthma; however, no therapeutic agent has demonstrated these findings in individuals with low Th2 inflammation.

What differentiates tezepelumab from its predecessors is an ability to treat endotypes with limited biological therapy options such as Th2-low asthma or uncontrolled allergic asthma with elevated IgE levels outside the dosing range of available biologics. Given that tezepelumab's mechanism of action is further upstream in the inflammatory cascade and central in modifying type 2 immunity, it also blocks the release of inflammatory mediators involved in type 1 inflammation such as innate lymphoid cells, neutrophils, mast cells, natural killer T cells and basophils, most likely inhibiting Th2-low inflammation pathways that have not been elucidated yet. ${ }^{24}$

Future research should further focus on tezepelumab's efficacy in the treatment of specific Th2-low asthma endotypes. Additional benefit may be derived from identifying biomarkers to identify which patients with Th2-low inflammation are most likely to respond to therapy with tezepelumab. Likewise, it is worth investigating if tezepelumab is beneficial in the treatment of syndromes associated with asthma and systemic inflammation such as obesity, allergic bronchopulmonary aspergillosis or eosinophilic granulomatous with polyangiitis given its unique mechanism of action.

In addition to the PATHFINDER program results, full results of SOURCE are warranted to further assess if tezepelumab recipients are able to reduce daily chronic oral corticosteroids or discontinue corticosteroids in a subset of uncontrolled asthma patients. If these studies conclude tezepelumab induces a difference in chronic oral corticosteroid cessation versus current standard of care this would demonstrate another unique indication for the monoclonal antibody. ${ }^{33}$ Lastly, the safety and efficacy of tezepelumab after one year of therapy necessitates further investigation.

\section{Abbreviations}

ICS, inhaled corticosteroids; Th, T-helper type; IL, interleukin; GM-CSF, granulocyte-macrophage colony-stimulating factor; IgE, immunoglobulin; TSLP, thymic stromal lymphopoietin; TNF- $\alpha$, tumor necrosis factor; NF- $\kappa B$, nuclear factor kappa-light chain-enhancer; RSV, respiratory syncytial virus; FE $E_{N O}$, fractional exhaled nitric oxide; $\mathrm{FEV}_{1}$, forced expiratory volume in 1 second; LABA, long-acting beta-agonist; AAER, annualized asthma exacerbation rate; 
ACQ, Asthma Control Questionnaire; AQLQ, Asthma Quality of Life Questionnaire; TARC, thymus and activation-regulated chemokine; BMI, body mass index.

\section{Author Contributions}

All authors made substantial contributions to conception and design, acquisition of data, or analysis and interpretation of data; took part in drafting the article or revising it critically for important intellectual content; agreed to submit to the current journal; gave final approval of the version to be published; and agree to be accountable for all aspects of the work.

\section{Disclosure}

Dr. Shenoy has performed advisory work on behalf of AstraZeneca. The authors report no other conflicts of interest in this work.

\section{References}

1. To T, Stanojevic S, Moores G, et al. Global asthma prevalence in adults: findings from the cross-sectional world health survey. $B M C$ Public Health. 2012;12(1):204. doi:10.1186/1471-2458-12-204

2. Backman H, Jansson S, Stridsman C, et al. Severe asthma-a population study perspective. Clin Exp Allergy. 2019;49(6):819-828. doi:10.1111/cea.13378

3. Busse WW, Holgate S, Kerwin E, et al. Randomized, double-blind, placebo-controlled study of brodalumab, a human anti-IL-17 receptor monoclonal antibody, in moderate to severe asthma. Am J Respir Crit Care Med. 2013;188(11):1294-1302. doi:10.1164/rccm.201212-2318OC

4. Anaptysbio reports top-line data from interim analysis of eclipse phase 2 clinical trial of etokimab in chronic rhinosinusitis with nasal polyps; 2020. Available from: https://www.globenewswire. com/Website. Accessed September 13, 2020.

5. Peters MC, Mekonnen ZK, Yuan S, Bhakta NR, Woodruff PG, Fahy JV. Measures of gene expression in sputum cells can identify TH2-high and TH2-low subtypes of asthma. J Allergy Clin Immunol. 2014;133(2):388-394. doi:10.1016/j.jaci.2013.07.036

6. Seys SF, Scheers H, Van den Brande P, et al. Cluster analysis of sputum cytokine-high profiles reveals diversity in $\mathrm{T}(\mathrm{h}) 2$-high asthma patients. Respir Res. 2017;18(1):39. doi:10.1186/s12931-017-0524-y

7. Wenzel SE, Schwartz LB, Langmack EL, et al. Evidence that severe asthma can be divided pathologically into two inflammatory subtypes with distinct physiologic and clinical characteristics. Am J Respir Crit Care Med. 1999;160(3):1001-1008. doi:10.1164/ajrccm.160.3.9 812110

8. Murdoch JR, Lloyd CM. Chronic inflammation and asthma. Mutat Res. 2010;690(1-2):24-39. doi:10.1016/j.mrfmmm.2009.09.005

9. Voehringer D, Reese TA, Huang X, Shinkai K, Locksley RM. Type 2 immunity is controlled by IL-4/IL-13 expression in hematopoietic non-eosinophil cells of the innate immune system. $J$ Exp Med. 2006;203(6):1435-1446. doi:10.1084/jem.20052448

10. Steinke JW, Borish L. Th2 cytokines and asthma. interleukin-4: its role in the pathogenesis of asthma, and targeting it for asthma treatment with interleukin-4 receptor antagonists. Respir Res. 2001;2 (2):66-70. doi:10.1186/rr40

11. Rabe KF, Nair P, Brusselle G, et al. Efficacy and safety of dupilumab in glucocorticoid-dependent severe asthma. N Engl J Med. 2018;378 (26):2475-2485. doi:10.1056/NEJMoa1804093
12. Castro M, Corren J, Pavord ID, et al. Dupilumab efficacy and safety in moderate-to-severe uncontrolled asthma. $N$ Engl J Med. 2018;378 (26):24862496. doi:10.1056/NEJMoa1804092

13. Demehri S, Morimoto M, Holtzman MJ, Kopan R, Liu Y-J. Skinderived TSLP triggers progression from epidermal-barrier defects to asthma. PLoS Biol. 2009;7(5):e1000067. doi:10.1371/journal. pbio. 1000067

14. Roan F, Bell BD, Stoklasek TA, Kitajima M, Han H, Ziegler SF. The multiple facets of thymic stromal lymphopoietin (TSLP) during allergic inflammation and beyond. $J$ Leukoc Biol. 2012;91 (6):877-886. doi:10.1189/jlb.1211622

15. Kool M, Willart MA, van Nimwegen M, et al. An unexpected role for uric acid as an inducer of $\mathrm{T}$ helper 2 cell immunity to inhaled antigens and inflammatory mediator of allergic asthma. Immunity. 2011;34(4):527-540. doi:10.1016/j.immuni.2011.03.015

16. Varricchi G, Pecoraro A, Marone G, et al. Thymic stromal lymphopoietin isoforms, inflammatory disorders, and cancer. Front Immunol. 2018;9:1595. doi:10.3389/fimmu.2018.01595

17. Roan F, Obata-Ninomiya K, Ziegler SF. Epithelial cell-derived cytokines: more than just signaling the alarm. $J$ Clin Invest. 2019;129 (4): 14411451 . doi: $10.1172 /$ jci124606

18. Ying S, O'Connor B, Ratoff J, et al. Expression and cellular provenance of thymic stromal lymphopoietin and chemokines in patients with severe asthma and chronic obstructive pulmonary disease. $J$ Immunol. 2008;181(4):2790-2798. doi:10.4049/jimmunol.1 81.4.2790

19. Ying S, O'Connor B, Ratoff J, et al. Thymic stromal lymphopoietin expression is increased in asthmatic airways and correlates with expression of Th2-attracting chemokines and disease severity. $J$ Immunol. 2005;174(12):81838190. doi:10.4049/jimmunol.17 4.12.8183

20. Shikotra A, Choy DF, Ohri CM, et al. Increased expression of immunoreactive thymic stromal lymphopoietin in patients with severe asthma. J Allergy Clin Immunol. 2012;129(1):104-109. doi:10.1016/j.jaci.2011.08.03

21. West EE, Kashyap M, Leonard WJ. TSLP: a key regulator of asthma pathogenesis. Drug Discov Today Dis Mech. 2012;9(3-4). doi:10.1016/j.ddmec.2012.09.003

22. Pham TH, Ren P, Parnes JR, et al. Tezepelumab reduces multiple key inflammatory biomarkers in patients with severe, uncontrolled asthma in the phase 2b PATHWAY study. Am J Respir Crit Care Med. 2019;199.

23. Gauvreau GM, O'Byrne PM, Boulet L, et al. Effects of an anti-TSLP antibody on allergen-induced asthmatic responses. $N$ Engl $J$ Med. 2014;370(22):2102-2110. doi:10.1056/NEJMoa1402895

24. Corren J, Parnes JR, Wang L, et al. Tezepelumab in adults with uncontrolled asthma. $N$ Engl $J$ Med. 2017;377(10):936-946. doi:10.1056/NEJMoa1704064

25. Corren J, Garcia E, Parnes JR, et al. Tezepelumab treatment effect on annualized rate of exacerbations by baseline biomarkers in uncontrolled severe asthma patients: phase $2 \mathrm{~b}$ PATHWAY study. Am J Respir Crit Care Med. 2019;199:199.

26. Corren J, Chen S, Callan L, et al. The impact of tezepelumab on hospitalization and emergency department visits in patients with severe uncontrolled asthma: results from the pathway phase $2 \mathrm{~b}$ trial. Am J Respir Crit Care Med. 2019;199.

27. Pham T, Ren P, Parnes JR, Griffiths JM. Tezepelumab reduces multiple key inflammatory biomarkers in patients with severe, uncontrolled asthma in the phase $2 \mathrm{~b}$ PATHWAY study. In: B21. Severe Asthma: Clinical and Mechanistic Studies. American Thoracic Society; 2019:A2677.

28. Ambrose C, Colice G, Salapa K, Parnes J, Corren J. Effect of tezepelumab on exacerbations in patients with severe, uncontrolled asthma, according to baseline body mass index: results from the phase 2b PATHWAY study. J Allergy Clin Immunol. 2020;145(2): AB25. doi:10.1016/j.jaci.2019.12.804 
29. Sridhar S, Zhao W, Pham T, et al. Tezepelumab decreases matrix remodeling and inflammatory pathways in patients with asthma. Eur Respir J. 2019;54(suppl63):RCT3785.

30. Menzies-Gow A, Colice G, Griffiths JM, et al. NAVIGATOR: a phase 3 multicentre, randomized, double-blind, placebo-controlled, parallel-group trial to evaluate the efficacy and safety of tezepelumab in adults and adolescents with severe, uncontrolled asthma. Respir Res. 2020;21(1):266. doi:10.1186/s12931-020-01526-6

31. Tezepelumab NAVIGATOR phase III trial met primary endpoint of a statistically significant and clinically meaningful reduction in exacerbations in a broad population of patients with severe asthma; 2020. Available from: https://www.astrazeneca.com/Website. Accessed December 7, 2020.
32. Update on SOURCE phase III trial for tezepelumab in patients with severe, oral corticosteroid-dependent asthma. Available from: https:// www.astrazeneca.com/content/astraz/media-centre/press-releases/ 2020/update-on-source-phase-iii-trial-for-tezepelumab-in-patientswith-severe-oral-corticosteroid-dependent-asthma.html. Accessed January 20, 2021.

33. Bel EH, Wenzel SE, Thompson PJ, et al. Oral glucocorticoid-sparing effect of mepolizumab in eosinophilic asthma. $N$ Engl $J$ Med. 2014;371(13):11891197. doi:10.1056/NEJMoa1403291

\section{Publish your work in this journal}

Drug Design, Development and Therapy is an international, peerreviewed open-access journal that spans the spectrum of drug design and development through to clinical applications. Clinical outcomes, patient safety, and programs for the development and effective, safe, and sustained use of medicines are a feature of the journal, which has also been accepted for indexing on PubMed Central. The manuscript management system is completely online and includes a very quick and fair peer-review system, which is all easy to use. Visit http://www. dovepress.com/testimonials.php to read real quotes from published authors. 